\title{
KINEMATICS OF A RIGID ABOUT A FIXED POINT
}

\author{
Do Sanh, Do Dang Khoa \\ Hanoi University of Hanoi
}

\begin{abstract}
This paper employs transmission matrices to investigate the motion of a rigid body about a fixed point. Formulas to calculate coordinates, velocities, and the accelerations of points on the body are presented. The method separates generalized velocities and accelerations making it particularly useful to the study of a wide range of problems in dynamics such as, for example, the calculation of the inertia matrix.
\end{abstract}

\section{INTRODUCTION}

The spatial motion of a rigid body is of interest in many problems in mechanics having application in gyroscopic motion, robotics, and mechanical processing. In particular, rigid body kinematics are of special interest to aerospace and shipping applications. Reference [2] presents a systematic investigation of this general subject though the methods used are complex. This paper describes the use of transmission matrices $[5,6]$ as a simple but powerful approach with the added advantage of being in a form well suited for use with the Mathcad and Mathlab software packages.

\section{BASIC THEORY}

In order to investigate the spatial motion of a rigid body two kirids of coordinate systems are required: one is a fixed frame of reference (ground-coordinate system) and the other is a system rigidly connected to the moving body (body-coordinate system).

It is well known that the general motion of a rigid body about a fixed point can be treated as a combination of rotations about a set of axes.

1) Rotation through angle $\varphi$ about the $x$-axis with corresponding matrix $T_{1}$ :

$$
\mathbf{T}_{1}=\left\|\begin{array}{ccc}
1 & 0 & 0 \\
0 & c \varphi & -s \varphi \\
0 & s \varphi & c \varphi
\end{array}\right\| .
$$

2) Rotation through angle $\psi$ about the $y$-axis with corresponding matrix $\mathrm{T}_{2}$ :

$$
\mathrm{T}_{2}=\left\|\begin{array}{ccc}
c \psi & 0 & s \psi \\
0 & 1 & 0 \\
-s \psi & 0 & c \psi
\end{array}\right\| .
$$

3) Rotation through angle $\theta$ about the $z$-axis with corresponding matrix $\mathrm{T}_{3}$

$$
\mathbf{T}_{3}=\left\|\begin{array}{ccc}
c \theta & -s \theta & 0 \\
s \theta & c \theta & 0 \\
0 & 0 & 1
\end{array}\right\| .
$$


For convenience, we write: $\cos (\varphi) \equiv C \varphi ; \sin (\varphi) \equiv S \varphi ; \cos (\varphi+\psi) \equiv C(\varphi+\psi), \ldots$ and denote matrices in bold type. All vectors are treated as $(3 \times 1)$ matrices.

The motion of a body about a fixed point can be performed by means of successive rotations about appropriate axes corresponding to above mentioned matrixes. Note that in general the axes need not be mutually perpendicular. Although many methods exist to investigate the motion of a body about a fixed point, this paper addresses only two of these, namely Euler Angles and the familiar Roll-Pitch-Yaw conventions used to describe the motion of ships and aircraft.

\subsection{Euler Angles}

Euler angles involve three successive rotations about three axes. In Fig. 1, denote the ground coordinate system by $O \xi \eta \zeta$ and the body coordinate system by $O x y z$, where ON (called the line of nodes) is the line of intersection of the $(x, y)$-plane with the $(\xi, \eta)$-plane $[2,4]$. The position of the body is defined by means of the three Euler angles $\varphi, \psi, \theta$, called respectively the pure, notation and procession angles. Let the two coordinate systems coincide at start time $t_{0}$ and denote the position of the body at later time $t$ as $O x y z$. It is possible to show that three successive rotations of the body about appropriate axes is sufficient to move the body from position $O x y z$ at time $t$ to the start position $O \xi \eta \zeta$.

One can proceed as follows

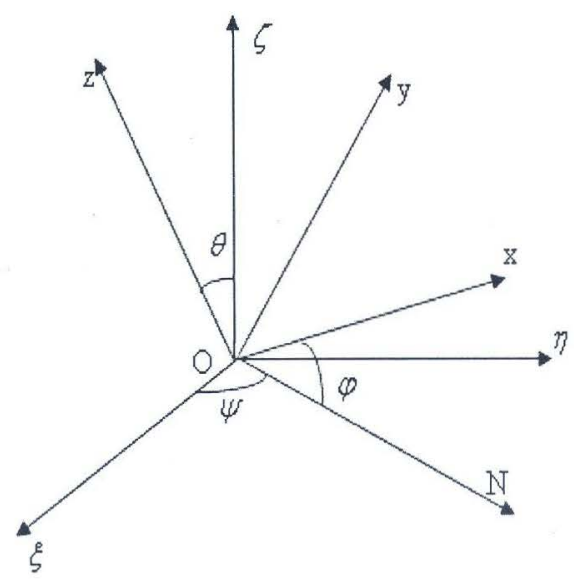

Fig. 1. Euler Angles

1) Rotate the body coordinate system $O x y z$ through angle $\varphi$ about the $z$-axis using $\mathbf{T}_{1}$

$$
\mathrm{T}_{1}=\left\|\begin{array}{ccc}
c \varphi & -s \varphi & 0 \\
s \varphi & c \varphi & 0 \\
0 & 0 & 1
\end{array}\right\|
$$

The body is now located at $O x_{1} y_{1} z_{1}$ and $O z_{1} \equiv O z, O x_{1} \equiv O N, O y_{1}$. 
2) Rotate the $O x_{1} y_{1} z_{1}$ coordinate system through angle $\theta$ about $\mathrm{ON}$ using $\mathbf{T}_{2}$

$$
\mathbf{T}_{2}=\left\|\begin{array}{ccc}
1 & 0 & 0 \\
0 & c \theta & -s \theta \\
0 & s \theta & c \theta
\end{array}\right\|
$$

The $O x_{1} y_{1} z_{1}$ body-coordinate system replaces $O x_{2} y_{2} z_{2}$ such that $O x_{2} \equiv O N, O z_{2} \equiv$ $\mathrm{O}_{\varsigma}, \mathrm{Oy}_{2}$.

3) Rotate the $O x_{2} y_{2} z_{2}$-body coordinate system about the $\mathrm{Oz}_{2}(\equiv O \varsigma)$ through $\psi$ using $\mathbf{T}_{3}$

$$
\mathbf{T}_{3}=\left\|\begin{array}{ccc}
c \psi & -s \psi & 0 \\
s \psi & c \psi & 0 \\
0 & 0 & 1
\end{array}\right\|
$$

The three successive rotations restore the body to the start position. The total displacement carried out in a single operation is accomplished using $\mathrm{T}$ :

$$
\begin{aligned}
\mathbf{T}=\mathbf{T}_{3} \mathbf{T}_{2} \mathbf{T}_{1} & =\left\|\begin{array}{ccc}
c \psi & -s \psi & 0 \\
s \psi & c \psi & 0 \\
0 & 0 & 1
\end{array}\right\|\left\|\begin{array}{ccc}
1 & 0 & 0 \\
0 & c \theta & -s \theta \\
0 & s \theta & c \theta
\end{array}\right\|\left\|\begin{array}{ccc}
c \varphi & -s \varphi & 0 \\
s \varphi & c \varphi & 0 \\
0 & 0 & 1
\end{array}\right\| \\
& =\left\|\begin{array}{ccc}
c \psi c \varphi-s \psi s \varphi c \theta & -s \varphi c \psi-s \psi c \varphi c \theta & s \psi s \theta \\
s \psi c \varphi+c \psi s \varphi c \theta & -s \varphi s \psi+c \psi c \varphi c \theta & -c \psi s \theta \\
s \theta s \varphi & s \theta c \varphi & c \theta
\end{array}\right\|
\end{aligned}
$$

The $\mathrm{T}$ matrix makes it also possible to determine the components of a vector rigidly connected to the body in the fixed coordinate system. In other words, a vector with components $(a, b, c)$ in body-coordinates will have components $(\xi, \eta, \zeta)$ in the fixed system related by:

$$
\left\|\begin{array}{l}
\xi \\
\eta \\
\zeta
\end{array}\right\|=\mathrm{T}_{3} \mathrm{~T}_{2} \mathrm{~T}_{1}\left\|\begin{array}{l}
a \\
b \\
c
\end{array}\right\|=\left\|\begin{array}{ccc}
c \varphi c \psi-s \varphi s \psi c \theta & -s \varphi c \psi-c \varphi s \psi c \theta & s \psi s \theta \\
c \varphi s \psi+s \varphi c \psi c \theta & -s \varphi s \psi+c \varphi c \psi c \theta & -c \psi s \theta \\
s \varphi s \theta & c \varphi s \theta & c \theta
\end{array}\right\|\left\|\begin{array}{l}
a \\
b \\
c
\end{array}\right\|
$$

Putting this another way,

$$
\mathrm{r}_{o}=\operatorname{Tr}
$$

where $\mathbf{r}$ and $\mathrm{r}_{o}$ are the respective position vectors of the point $M$ connected rigidly to the body -coordinate and fixed coordinate systems. The inverse displacement is accomplished using T':

$$
\begin{aligned}
\mathrm{T}^{\prime} & =\left\|\begin{array}{ccc}
c \varphi & s \varphi & 0 \\
-s \varphi & c \varphi & 0 \\
0 & 0 & 1
\end{array}\right\|\left\|\begin{array}{ccc}
c \theta & s \theta & 0 \\
-s \theta & c \theta & 0 \\
0 & 0 & 1
\end{array}\right\|\left\|\begin{array}{ccc}
c \psi & s \psi & 0 \\
-s \psi & c \psi & 0 \\
0 & 0 & 1
\end{array}\right\| \\
& =\left\|\begin{array}{ccc}
c \varphi c \psi-s \varphi s \psi c \theta & c \varphi s \psi+s \varphi c \psi c \theta & s \varphi s \theta \\
-s \varphi c \psi-c \varphi s \psi c \theta & -s \varphi s \psi+c \varphi c \psi c \theta & c \varphi s \theta \\
s \psi s \theta & -c \psi s \theta & c \theta
\end{array}\right\|
\end{aligned}
$$

As above, write

$$
\mathrm{r}=\mathrm{T}^{\prime} \mathrm{r}_{o}
$$

The matrices $\mathrm{T}^{\prime}$ and $\mathbf{T}$ are orthogonal, so that $\mathrm{TT}^{\prime}=\mathrm{I}$. 
Expression (2) makes it possible to calculate the velocity of a point anywhere on the body.

To so this, introduce the following matrices:

$$
\begin{aligned}
& \mathbf{T}_{11}=\left\|\begin{array}{ccc}
-s \varphi & -c \varphi & 0 \\
c \varphi & -s \varphi & 0 \\
0 & 0 & 0
\end{array}\right\| ; \quad \mathbf{T}_{22}=\left\|\begin{array}{ccc}
0 & 0 & 0 \\
0 & -s \theta & -c \theta \\
0 & c \theta & -s \theta
\end{array}\right\| ; \quad \mathbf{T}_{33}=\left\|\begin{array}{ccc}
-s \psi & -c \psi & 0 \\
c \psi & -s \psi & 0 \\
0 & 0 & 0
\end{array}\right\| \\
& \mathbf{r}_{1}=\left\|\begin{array}{c}
a \dot{\varphi} \\
b \dot{\varphi} \\
c \dot{\varphi}
\end{array}\right\| ; \quad \mathbf{r}_{2}=\left\|\begin{array}{c}
a \dot{\theta} \\
b \dot{\theta} \\
c \dot{\theta}
\end{array}\right\| ; \quad \mathbf{r}_{3}=\left\|\begin{array}{c}
a \dot{\psi} \\
b \dot{\psi} \\
c \dot{\psi}
\end{array}\right\|
\end{aligned}
$$

The velocity of the point on the body is computed from:

$$
\mathrm{v}=\left\|\begin{array}{c}
\dot{\xi} \\
\dot{\eta} \\
\dot{\zeta}
\end{array}\right\|=\mathrm{T}_{33} \mathrm{~T}_{2} \mathrm{~T}_{1} \mathrm{r}_{3}+\mathrm{T}_{3} \mathrm{~T}_{22} \mathrm{~T}_{1} \mathrm{r}_{2}+\mathrm{T}_{3} \mathrm{~T}_{2} \mathrm{~T}_{11} \mathrm{r}_{1}
$$

Expression (7) separates out the generalized velocity. Note for example, the term including the generalized velocity $\dot{\theta}$ is:

$$
\begin{aligned}
\mathrm{T}_{3} \mathrm{~T}_{22} \mathrm{~T}_{1} \mathbf{r}_{2} & =\left\|\begin{array}{ccc}
c \psi & -s \psi & 0 \\
s \psi & c \psi & 0 \\
0 & 0 & 1
\end{array}\right\|\left\|\begin{array}{ccc}
-s \theta & -c \theta & 0 \\
c \theta & -s \theta & 0 \\
0 & 0 & 0
\end{array}\right\|\left\|\begin{array}{ccc}
c \varphi & -s \varphi & 0 \\
s \varphi & c \varphi & 0 \\
0 & 0 & 1
\end{array}\right\|\left\|\begin{array}{c}
a \dot{\theta} \\
b \dot{\theta} \\
\dot{\theta}
\end{array}\right\| \\
& =\left\|\begin{array}{ccc}
s \varphi s \psi s \theta & c \varphi s \psi s \theta & s \psi c \theta \\
-s \varphi c \psi s \theta & -c \varphi c \psi s \theta & -c \psi c \theta \\
s \varphi c \theta & c \varphi c \theta & -s \theta
\end{array}\right\|\left\|\begin{array}{c}
a \dot{\theta} \\
b \dot{\theta} \\
c \dot{\theta}
\end{array}\right\|
\end{aligned}
$$

which in turn yields the following expressions for the generalized velocity $\dot{\theta}$ :

$$
\begin{aligned}
& \dot{\xi}(\dot{\theta})=(a s \varphi s \psi s \theta+b c \varphi s \psi s \theta+c s \psi c \theta) \dot{\theta} ; \\
& \dot{\eta}(\dot{\theta})=-(a s \varphi c \psi s \theta+b c \varphi c \psi s \theta+c \psi c \theta) \dot{\theta} ; \\
& \dot{\zeta}(\dot{\theta})=(a s \varphi c \theta+b c \varphi c \theta+c s \theta) \dot{\theta}
\end{aligned}
$$

To obtain the attendant accelerations, introduce the following matrices:

$$
\begin{gathered}
\mathrm{T}_{111}=\left\|\begin{array}{ccc}
-c \varphi & s \varphi & 0 \\
-s \varphi & -c \varphi & 0 \\
0 & 0 & 0
\end{array}\right\| ; \mathbf{T}_{222}=\left\|\begin{array}{ccc}
0 & 0 & 0 \\
0 & -c \theta & s \theta \\
0 & 0 & 0
\end{array}\right\| ; \mathrm{T}_{\mathbf{3 3 3}}=\left\|\begin{array}{ccc}
-c \psi & s \psi & 0 \\
-s \psi & -c \psi & 0 \\
0 & 0 & 0
\end{array}\right\| \\
\mathrm{r}_{11}=\left\|\begin{array}{c}
a \ddot{\varphi} \\
b \ddot{\varphi} \\
c \ddot{\varphi}
\end{array}\right\| ; \quad \mathbf{r}_{22}=\left\|\begin{array}{c}
a \ddot{\theta} \\
b \ddot{\theta} \\
c \ddot{\theta}
\end{array}\right\| ; \quad \mathbf{r}_{33}=\left\|\begin{array}{c}
a \ddot{\psi} \\
b \ddot{\psi} \\
c \ddot{\psi}
\end{array}\right\| ; \\
\mathrm{r}_{1,1}=\left\|\begin{array}{c}
a \dot{\varphi}^{2} \\
b \dot{\varphi}^{2} \\
c \dot{\varphi}^{2}
\end{array}\right\| ; \mathbf{r}_{2,2}=\left\|\begin{array}{c}
a \dot{\theta}^{2} \\
b \dot{\theta}^{2} \\
c \dot{\theta}^{2}
\end{array}\right\| ; \mathbf{r}_{3,3}=\left\|\begin{array}{c}
a \dot{\psi}^{2} \\
b \dot{\psi}^{2} \\
c \dot{\psi}^{2}
\end{array}\right\|
\end{gathered}
$$




$$
\mathrm{r}_{1,2}=\mathrm{r}_{2,1}=\left\|\begin{array}{c}
a \dot{\varphi} \dot{\theta} \\
b \dot{\varphi} \dot{\theta} \\
c \dot{\varphi} \dot{\theta}
\end{array}\right\| ; \mathrm{r}_{1,3}=\mathrm{r}_{3,1}=\left\|\begin{array}{c}
a \dot{\varphi} \dot{\psi} \\
b \dot{\varphi} \dot{\psi} \\
c \dot{\varphi} \dot{\psi}
\end{array}\right\| ; \mathrm{r}_{2,3}=\mathrm{r}_{3,2}=\left\|\begin{array}{c}
a \dot{\theta} \dot{\psi} \\
b \dot{\theta} \dot{\psi} \\
c \dot{\theta} \dot{\psi}
\end{array}\right\|
$$

The accelerations of a point on the body are written in the form:

$$
\begin{aligned}
& \mathrm{a}=\left\|\begin{array}{c}
\ddot{\xi} \\
\ddot{\eta} \\
\ddot{\zeta}
\end{array}\right\|=\mathrm{T}_{33} \mathrm{~T}_{2} \mathrm{~T}_{1} \mathrm{r}_{33}+\mathrm{T}_{3} \mathrm{~T}_{22} \mathrm{~T}_{1} \mathrm{r}_{22}+\mathrm{T}_{3} \mathrm{~T}_{2} \mathrm{~T}_{11} \mathrm{r}_{11}+\mathrm{T}_{333} \mathrm{~T}_{2} \mathrm{~T}_{1} \mathrm{r}_{3,3} \\
& +\mathrm{T}_{3} \mathrm{~T}_{222} \mathrm{~T}_{1} \mathrm{r}_{2,2}+\mathrm{T}_{3} \mathrm{~T}_{2} \mathrm{~T}_{111} \mathrm{r}_{1,1}+2 \mathrm{~T}_{33} \mathrm{~T}_{22} \mathrm{~T}_{1} \mathrm{r}_{2,3} \\
& +2 \mathrm{~T}_{33} \mathrm{~T}_{2} \mathrm{~T}_{11} \mathrm{r}_{1,3}+2 \mathrm{~T}_{3} \mathrm{~T}_{22} \mathrm{~T}_{11} \mathrm{r}_{1,2}
\end{aligned}
$$

The transmission matrices $\mathbf{T}$ and $\mathbf{T}^{\prime}$ allow us to determine the angular velocity of the body in the body-coordinate and fixed coordinate systems. Denote the angular velocity in the body-coordinate and the fixed coordinate systems respectively as $\omega$ and $\omega^{\mathrm{o}}$. Applying (3) and (5) we have

$$
\begin{aligned}
& \omega^{\mathbf{o}}=\mathrm{T} \omega \\
& \omega=\mathrm{T}^{\prime} \omega^{\mathrm{o}}
\end{aligned}
$$

To illustrate the process, determine the angular velocity of the body in the fixed coordinate system and apply the theorem for the addition of angular velocities, we get:

$\boldsymbol{\Omega}=\dot{\varphi}+\dot{\boldsymbol{\theta}}+\dot{\psi}(\mathrm{a})$ where $\Omega$ is the absolute angular velocity of the body.

It is easy then to show: $\Omega \equiv \omega^{\circ}$

To apply (9), one must first calculate the components of the angular velocity in the body -coordinate system. First determine the components of $\dot{\psi}$ in the body-coordinate system using T' to calculate $\mathrm{T}^{\prime} \dot{\boldsymbol{\psi}}$ :

$$
\mathrm{T}^{\prime} \dot{\psi}=\left\|\begin{array}{ccc}
c \varphi c \psi-s \varphi s \psi c \theta & c \varphi s \psi+s \varphi c \psi+s \varphi c \psi c \theta & s \varphi s \theta \\
-s \varphi c \psi-c \varphi s \psi c \theta & -s \varphi s \psi+c \varphi c \psi c \theta & c \varphi s \theta \\
s \psi s \theta & -c \psi s \theta & c \theta
\end{array}\right\|\left\|\begin{array}{c}
0 \\
0 \\
\dot{\psi}
\end{array}\right\|=\left\|\begin{array}{c}
s \varphi s \theta \dot{\psi} \\
c \varphi s \theta \dot{\psi} \\
c \theta \dot{\psi}
\end{array}\right\|
$$

The components of the angular velocity of the body in the body-coordinate system become

$$
\omega=\left\|\begin{array}{l}
\dot{\psi} s \varphi s \theta+\dot{\theta} c \varphi \\
\dot{\psi} c \varphi s \theta-\dot{\theta} s \varphi \\
\dot{\psi} c \theta+\dot{\varphi}
\end{array}\right\|
$$

From (9) obtain:

$$
\begin{aligned}
\omega^{\mathbf{o}}=\mathbf{T} \omega & =\left\|\begin{array}{ccc}
c \varphi c \psi-s \varphi s \psi c \theta & -s \varphi c \psi-c \varphi s \psi c \theta & s \psi s \theta \\
c \varphi s \psi+s \varphi c \psi c \theta & -s \varphi s \psi+c \varphi c \psi c \theta & -c \psi s \theta \\
s \varphi s \theta & c \varphi s \theta & c \theta
\end{array}\right\|\left\|\begin{array}{c}
\dot{\psi} s \varphi s \theta+\dot{\theta} c \varphi \\
\dot{\psi} c \varphi s \theta-\dot{\theta} s \varphi \\
\dot{\psi} c \theta+\dot{\varphi}
\end{array}\right\| \\
& =\left\|\begin{array}{c}
\dot{\varphi} s \psi s \theta+\dot{\theta} c \psi \\
-\dot{\varphi} c \psi s \theta+\dot{\theta} s \psi \\
\dot{\varphi} c \theta+\dot{\psi}
\end{array}\right\|
\end{aligned}
$$

As an example of how to apply the method, consider the gyroscope shown in Fig. 2. 




Fig. 2. Equilibrium Gyroscope

Assume the disk rotates about the horizontal axis CD with constant angular velocity $\omega_{1}$ and CD in turn rotates about the vertical axis with constant angular velocity $\omega_{2}$. The bodycoordinate and fixed coordinate systems are as shown. Clearly, $\psi \equiv 0, \varphi=\omega_{1} t, \theta=\omega_{2} t$ in which case the angular velocity of the body in the body-coordinate system is:

$$
\omega=\left\|\begin{array}{l}
\omega_{2} \cos \omega_{1} t \\
\omega_{2} \sin \omega_{1} t \\
\omega_{1}
\end{array}\right\|
$$

Matrix $\mathbf{T}$ then becomes

$$
\mathbf{T}=\left\|\begin{array}{ccc}
\cos \omega_{1} t & -\sin \omega_{1} t & 0 \\
\sin \omega_{1} t \cos \omega_{2} t & \cos \omega_{1} t \cos \omega_{2} t & \cos \omega_{2} t \\
0 & 0 & 1
\end{array}\right\|
$$

The angular velocity of the body in the fixed coordinate system becomes

$$
\omega^{o}=\Gamma \omega=\left\|\begin{array}{c}
0 \\
\omega_{2} \cos \omega_{2} t-\omega_{1} \sin \omega_{2} t \\
\omega_{2} \sin \omega_{2} t+\omega_{1} \cos \omega_{2} t
\end{array}\right\|
$$

\subsection{Roll-Pitch-Yaw Angles}

The shipping and aerospace industries adopt the Roll-Pitch-Yaw convention [2]. Direct the $\eta$-axis to point up and draw the other axes while observing the right-hand rule. In an aircraft body coordinate system the $x y$-plane coincides with the plane of symmetry of the aircraft, and the $x$-axis points from tail to cabin. The $y$-axis points up.

To determine the yaw, pitching and rolling angles, draw lines $\mathrm{ON}_{1}, \mathrm{ON}_{2}, \mathrm{ON}_{3}$, where $\mathrm{ON}_{1}$ is the intersection of the $(\xi \zeta)$-plane and the $(y, z)$-plane, $\mathrm{ON}_{2}$ is the intersection of the $(\xi \zeta)$-plane and the $(x, \eta)$-plane and $\mathrm{ON}_{3}$ is the intersection of the $(x, \eta)$-plane with the $(\xi, \eta)$-plane. Fig. 3 shows the yaw, pitching and rolling angles. Rigid body rotations are determined as follows:

1) Rotate the body through $\varphi$ about the $O x$ axis. Described by $\mathbf{T}_{1}$

$$
\mathbf{T}_{1}=\left\|\begin{array}{ccc}
1 & 0 & 0 \\
0 & c \varphi & -s \varphi \\
0 & s \varphi & c \varphi
\end{array}\right\|
$$




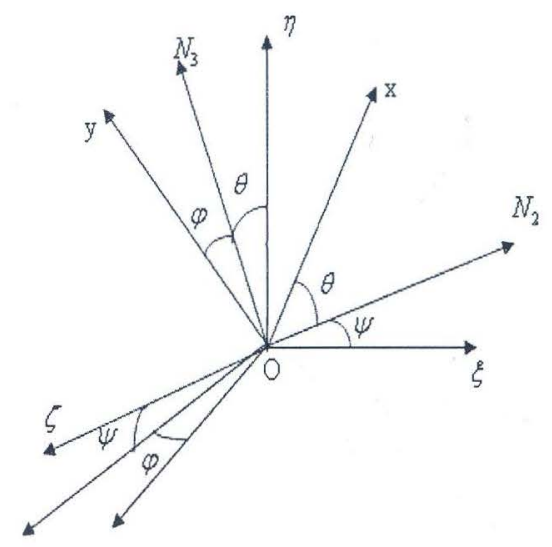

Fig. 3. Roll-Pitch-Yaw Angles

2) Rotate the body through $\theta$ about the $z$-axis. Described by $\mathbf{T}_{2}$

$$
\mathbf{T}_{2}=\left\|\begin{array}{ccc}
c \theta & -s \theta & 0 \\
s \theta & c \theta & 0 \\
0 & 0 & 1
\end{array}\right\|
$$

3) Rotate through $\psi$ about the $y$-axis. Described by $\mathbf{T}_{3}$

$$
\mathbf{T}_{3}=\left\|\begin{array}{ccc}
c \psi & 0 & s \psi \\
0 & 1 & 0 \\
-s \psi & 0 & c \psi
\end{array}\right\|
$$

The transmission matrix takes the form

$$
\begin{aligned}
\mathbf{T}=\mathbf{T}_{3} \mathbf{T}_{2} \mathbf{T}_{1} & =\left\|\begin{array}{ccc}
c \psi & 0 & s \psi \\
0 & 1 & 0 \\
-s \psi & 0 & c \psi
\end{array}\right\|\left\|\begin{array}{ccc}
c \theta & -s \theta & 0 \\
s \theta & c \theta & 0 \\
0 & 0 & 1
\end{array}\right\|\left\|\begin{array}{ccc}
1 & 0 & 0 \\
0 & c \varphi & -s \varphi \\
0 & s \varphi & c \varphi
\end{array}\right\| \\
& =\left\|\begin{array}{ccc}
c \psi c \theta & s \varphi s \psi+c \varphi c \psi s \theta & s \varphi c \psi s \theta-c \varphi s \psi \\
-s \theta & c \varphi c \theta & s \varphi c \theta \\
s \psi c \theta & c \varphi s \psi s \theta-s \varphi c \psi & c \varphi c \psi+s \varphi s \psi s \theta
\end{array}\right\|
\end{aligned}
$$

The inverse transmission matrix becomes

$$
\mathbf{T}^{\prime}=\left\|\begin{array}{ccc}
c \psi c \theta & s \theta & -s \psi c \theta \\
s \varphi s \psi-c \varphi c \psi s \theta & c \varphi c \theta & s \varphi c \psi+c \varphi s \psi s \theta \\
c \varphi s \psi+s \varphi c \psi s \theta & -s \varphi c \theta & c \varphi c \psi-s \varphi s \psi s \theta
\end{array}\right\|
$$

Krylov angles are used in shipping [2]. To construct the Oxyz-body coordinate system, orientate the Ox-axis from the ship's rudder to the bows, and direct the Oy-axis to the port (left) side. The $O z$-axis is in the diametrical plane of the ship.

To determine the Roll, Pitch and Yaw angles, draw lines $\mathrm{ON}_{1}, \mathrm{ON}_{2}, \mathrm{ON}_{3}$ Line $\mathrm{ON}_{1}$ is the intersection of the $(x, y)$ and $(\xi, \zeta)$ planes, and lines $\mathrm{ON}_{2}$ and $\mathrm{ON}_{3}$ are respectively perpendicular to $\mathrm{ON}_{1}$ in the $(x, y)$ plane and the $(\xi \zeta)$-side project plane. The rotations 


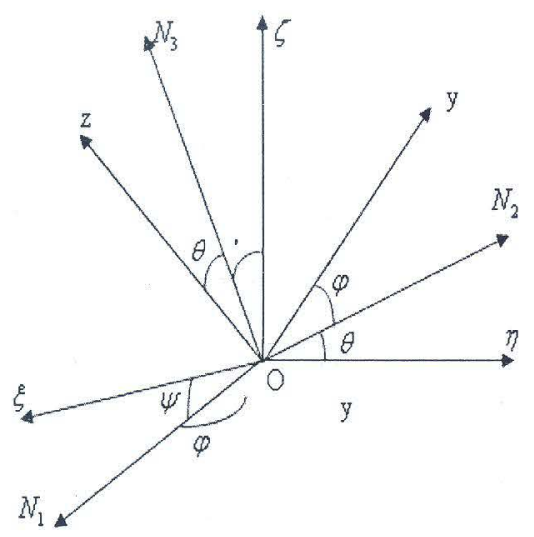

Fig. 4. Krylov Angles

about the axes $\mathrm{Oz}, \mathrm{Ox}$ and $\mathrm{O} \eta$ are denoted respectively: $\varphi, \theta$ and $\psi \cdot \mathbf{T}_{1}, \mathbf{T}_{2}$, and $\mathbf{T}_{3}$ are the corresponding rotation matrices:

$$
\mathbf{T}_{1}=\left\|\begin{array}{ccc}
c \varphi & -s \varphi & 0 \\
s \varphi & c \varphi & 0 \\
0 & 0 & 1
\end{array}\right\| ; \mathbf{T}_{2}=\left\|\begin{array}{ccc}
1 & 0 & 0 \\
0 & c \theta & -s \theta \\
0 & s \theta & c \theta
\end{array}\right\| ; \mathbf{T}_{3}=\left\|\begin{array}{ccc}
c \psi & 0 & s \psi \\
0 & 1 & 0 \\
-s \psi & 0 & c \psi
\end{array}\right\|
$$

The transmission matrix $\mathrm{T}$ takes the form

$$
\begin{aligned}
\mathbf{T}=\mathbf{T}_{\mathbf{3}} \mathbf{T}_{\mathbf{2}} \mathbf{T}_{\mathbf{1}} & =\left\|\begin{array}{ccc}
c \psi & 0 & s \psi \\
0 & 1 & 0 \\
-s \psi & 0 & c \psi
\end{array}\right\|\left\|\begin{array}{ccc}
1 & 0 & 0 \\
0 & c \theta & -s \theta \\
0 & s \theta & c \theta
\end{array}\right\|\left\|\begin{array}{ccc}
c \varphi & -s \varphi & 0 \\
s \varphi & c \varphi & 0 \\
0 & 0 & 1
\end{array}\right\| \\
& =\left\|\begin{array}{ccc}
c \varphi c \psi+s \varphi s \psi s \theta & c \varphi s \psi s \theta-s \varphi c \psi & s \psi c \theta \\
s \varphi c \theta & c \varphi c \theta & -s \theta \\
s \varphi c \psi s \theta-c \varphi s \psi & s \varphi s \psi+c \varphi c \psi s \theta & c \psi c \theta
\end{array}\right\|
\end{aligned}
$$

The inverse transmission matrix $T^{\prime}$ takes the form

$$
\begin{aligned}
\mathbf{T}^{\prime} & =\left\|\begin{array}{ccc}
c \varphi & s \varphi & 0 \\
-s \varphi & c \varphi & 0 \\
0 & 0 & 1
\end{array}\right\|\left\|\begin{array}{ccc}
1 & 0 & 0 \\
0 & c \theta & s \theta \\
0 & -s \theta & c \theta
\end{array}\right\|\left\|\begin{array}{ccc}
c \psi & 0 & -s \psi \\
0 & 1 & 0 \\
s \psi & 0 & c \psi
\end{array}\right\| \\
& =\left\|\begin{array}{ccc}
c \varphi c \psi+s \varphi s \psi s \theta & s \varphi c \theta & s \varphi c \psi s \theta-c \varphi s \psi \\
c \varphi s \psi s \theta-s \varphi c \psi & c \varphi c \theta & s \varphi s \psi+c \varphi c \psi s \theta \\
s \psi c \theta & -s \theta & c \psi c \theta
\end{array}\right\|
\end{aligned}
$$

Matrices $\mathbf{T}$ and $\mathbf{T}^{\prime}$ make it easy to determine the components of the angular velocity of the body in the body-coordinate and fixed coordinate systems. Working with Roll-PitchYaw angles similarly establishes formulae (7), (8), (9), and (10). 


\section{CONCLUSION}

The transmission matrix is used to investigate the kinematics of the rigid body about a fixed point. The proposed method is a general, simple and useful tool with which to conduct investigations into the motion of aircraft and ships, and other fields in which rigid body rotations play a significant role. It is clear method formulates problems in terms that are very convenient for general engineering use with special purpose software such as Mathcad, and Maple.

This work was completed with the financial support of the Basic Program in Natural Science.

\section{REFERENCES}

1. E. J. Haug, Computer Aided Kinematics and Dynamics of Mechanical systems, Vol. 1: Basic Methods. Allyn and Bacon, Boston 1989.

2. A. I. Lurie, Analytical Mechanics, Springer-Verlag, Berlin 2002.

3. P. E. Nikravesh, Computer Aided Analysis of Mechanical Systems, Prentice-Hall Englewood Cliffs, New Jersay, 1989

4. A. A. Shabana, Dynamics of Multi-body Systems, Willey ( $3^{\text {th }}$ Edition), Cambridge University Press, Cambridge, 2005.

5. Do Sanh, DoDang Khoa, The Method of Transmission Matrix for Investigating Planar Mechanisms, Vietnam Journal of Mechanics 29 (2007) (1).

6. Do Sanh, Do Dang Khoa, The method of transmission matrix for investigating planar relative motions, Vietnam Journal of Mechanics 29 (2) (2007) 105-116.

Received January 10, 2007

\section{KHẢO SÁT ĐỘNG HỌC CỦA VẬT RẮN QUAY QUANH MỘT ĐIỂM CỐ DỊNH}

Trong bài báo sử dụng phương pháp ma trận chuyền để khảo sát động học của vật rắn chuyển động quanh một điểm cố định. Trong phương pháp được đưa ra đã sử dụng các phương pháp: phương pháp góc Ole, phương pháp góc Roll-Pitch-Yaw. Đặc biệt trong các biểu thức tính vận tốc, tính gia tốc các diểm thuộc vật trong các công thức này cho phép xác định riêng rẽ các số hạng chứa các vận tốc suy rông, các gia tốc suy rộng.

Phương pháp được trình bày rất đơn giản, rất tiện lợi dối với các kỹ sư, các người làm kỹ thuật nhờ có thể sử dụng trực tiếp các phần mềm chuyên dụng như Mathcad, Maple, ... 